\title{
Challenges and opportunities of modelling carbon dioxide sequestration potential in Ukrainian soils
}

\author{
V. R. Cherlinka ${ }^{* 1,2}$, Y. M. Dmytruk 1 , V. I. Sobko ${ }^{3}$, M. V. Gunchak ${ }^{3}$, \\ T. I. Balan ${ }^{1}$, L. V. Cherlinka1 \\ ${ }^{1}$ Yuriy Fedkovych Chernivtsi National University, Chernivtsi, Ukraine \\ ${ }^{2}$ EOS Data Analytics inc, Menlo Park CA, USA \\ ${ }^{3}$ Chernivtsi branch of the State Institution "Soils Protection Institute of Ukraine", Chernivtsi, Ukraine
}

\begin{tabular}{|c|c|}
\hline ARTICLE INFO & ABSTRACT \\
\hline $\begin{array}{l}\text { Received } 06.11 .2021 \\
\text { Received in revised } \\
\text { form } 26.11 .2021 \\
\text { Accepted } 10.12 .2021 \\
\text { Available online } \\
20.12 .2021\end{array}$ & $\begin{array}{l}\text { The article examines approaches to modelling carbon dioxide sequestration by soils at } \\
\text { different scale levels and describes a number of problems encountered in this process. The } \\
\text { main problems in the modelling of organic carbon reserves for the territory of Ukraine have } \\
\text { been identified, among which: lack of high-resolution data (hard to access), including types } \\
\text { of land use; need for harmonization of existing data; the need for predicative modelling of } \\
\text { indicators (clay and organic carbon content) for areas where data are not available; } \\
\text { sianificant amounts of machine time for actual modelling The need for open access to }\end{array}$ \\
\hline $\begin{array}{l}\text { Keywords: } \\
\text { GSOCseq; } \\
\text { carbon dioxide } \\
\text { sequestration; } \\
\text { predicative algorithms; } \\
\text { modelling }\end{array}$ & $\begin{array}{l}\text { Also, the need to access large-scale topographic data as in the form of scanned topographic } \\
\text { maps M } 1: 10000 \text { and vectorized isolines are substantiated. It is shown that calculations of } \\
\text { areas based on rasters in the geographical coordinate system to ensure the same pixel size, } \\
\text { regardless of the location within Ukraine, it is proposed to use the "Ukraine Albers conic } \\
\text { equal area" projection based on the Datum Pulkovo } 1942 \text { for which the error in the lengths } \\
\text { of the lines is only } 0.1 \% \text {. Within areas can be used as a proposed projection, as well as } \\
\text { precise Gauss-Kruger projections (EPSG } 28404-28407,5565-5583) \text {. In this case, it is } \\
\text { necessary to take into account the likelihood of finding individual areas in several of the listed } \\
\text { zones. It is demonstrated that simulation allows to establish territories in which even the best } \\
\text { practices of management do not provide neutralization of emission of organic carbon from } \\
\text { soils. However, it is necessary to introduce a priority introduction of adaptive management } \\
\text { of the soil resources of agro-landscapes that will contribute to carbon sequestration or } \\
\text { minimization of its emission. }\end{array}$ \\
\hline
\end{tabular}

Citing: Cherlinka, V.R., Dmytruk, Y.M., Sobko, V.I., Gunchak, M.V., Balan, T. I. \& Cherlinka, L.V. (2021). Challenges and opportunities of modelling carbon dioxide sequestration potential in Ukrainian soils. AgroChemistry and Soil Science. 92, 62-70. doi:10.31073/acss92-07

\section{References}

1. United Nations, and Canada. (1992). United Nations Framework Convention on Climate Change. [New York]: United Nations, General Assembly,

2. Kyoto Protocol to the United Nations Framework Convention on Climate Change. (1997). Dec. 10, 1997. 2303 U.N.T.S. 162.

3. Paris Agreement to the United Nations Framework Convention on Climate Change.(2015) Dec. 12, 2015. T.I.A.S. No. 16-1104

4. Graham, F. (2021). COP26: Glasgow Climate Pact signed into history. Nature. DOI: https://doi.org/10.1038/d41586-021-03464-9.

5. IPCC. 2018. Global Warming of $1.5^{\circ} \mathrm{C}$. An IPCC Special Report on the impacts of global warming of $1.5^{\circ} \mathrm{C}$ above pre-industrial levels and related global greenhouse gas emission pathways, in the context of strengthening the global response to the threat of climate change, sustainable development, and efforts to eradicate poverty [Masson-Delmotte, V., P. Zhai, H.-O. Pörtner, D. Roberts, J. Skea, P.R. Shukla, A. Pirani, W. Moufouma-Okia, C. Péan, R. Pidcock, S. Connors, J.B.R. Matthews, Y. Chen, X. Zhou, M.I. Gomis, E. Lonnoy, T. Maycock, M. Tignor, and T. Waterfield (Eds.)].

6. Boincean, B. P. \& Dent, D. L. (2019). Farming the black earth. Sustainable and Climate-Smart Management of Chernozem Soil. Spring Nature, Cham, Switherland AG. doi: 10.1007/978-3-030-22533-9.

7. Lal, R. (2004). Soil carbon sequestration impacts on global climate change and food security. Science, 304(5677), 1623-1627. doi: 10.1126/science.1097396

8. Vargas-Rojas, R., Cuevas-Corona, R., Yigini Y., Tong, Y., Bazza, Z. \& Wiese L. (2018). International Yearbook of Soil Law and Policy. In Ginzky H., Dooley E., Heuser I.L., Kasimbazi E., Markus T., Qin T. (Eds.). International Yearbook of Soil Law and Policy. Cham, Springer International Publishing. pp. 373-395. doi: 10.1007/978-3-030-00758-4_18.

9. FAO. (2017). Unlocking the potential of soil organic carbon. Food and Agriculture Organization of the United Nations. Rome, Italy.

10. FAO. (2020). A protocol for measurement, monitoring, reporting and verification of soil organic carbon in agricultural landscapes GSOC-MRV Protocol. Rome. doi: 10.4060/cb0509en.

11. FAO. (2021). Global Soil Organic Carbon Sequestration Potential Map (GSOCseq). URL: https://www.fao.org/global-soilpartnership/gsocseq-map/en.

12. FAO. (2020). Technical specifications and country guidelines for Global Soil Organic Carbon Sequestration Potential Map (GSOCseq). Rome, Italy. URL: https://www.fao.org/documents/card/ru/c/cb0353en.

13. Jenkinson, D. S. \& Rayner, J. H. (1977). The turnover of soil organic matter in some of the Rothamsted classical experiments. Soil science, 123(5), 298-305. doi: 10.1097/00010694-197705000-00005.

14. Jenkinson, D. S. (1990). The turnover of organic carbon and nitrogen in soil. Philosophical Transactions of the Royal Society of London. Series B: Biological Sciences, 329, 361-368. doi: 10.1098/rstb.1990.0177.

15. Sierra, C. A., Müller, M. \& Trumbore, S. E. (2012). Models of soil organic matter decomposition: the SoilR package, version 1.0. Geoscientific Model Development, 5.4, 1045-1060. doi: 10.5194/gmd-5-1045-2012.

16. Coleman, K. \& Jenkinson, D. S. (1996). RothC-26.3-A Model for the turnover of carbon in soil. In: Powlson D.S., Smith P., Smith J.U. (Eds.). Evaluation of Soil Organic Matter Models. NATO ASI Series (Series I: Global Environmental Change). Vol. 38. Springer, Berlin, Heidelberg. doi: 10.1007/978-3-642-61094-3 17 .

17. Falloon, P. \& Smith, P. (2009). Modeling Soil Carbon Dynamics. In: Kutsch W. L., Bahn M., Heinemeyer A. (Eds.). Soil carbon dynamics: an integrated methodology. Cambridge University Press. doi: 10.1017/CBO9780511711794. 
18. Mitášová, H. \& Mitáš, L. (1993). Interpolation by regularized spline with tension: I. Theory and implementation. Mathematical Geology, 25(6), 641-655.

19. GRASS Development Team. Geographic Resources Analysis Support System (GRASS GIS) Software. (2021). Version 7.8.6. Open Source Geospatial Foundation, 2021. URL: http://grass.osgeo.org (online; accessed: 1.09.2021).

20. R Development Core Team. (2021). R: A language and environment for statistical computing. R Foundation for Statistical Computing. URL: http://www.r-project.org.

21. Breiman, L., (2001). Random forests. Machine learning, 45(1), 5-32. doi: 10.1023/A:1010933404324.

22. NASA JPL. (2013). NASA Shuttle Radar Topography Mission Global 1 arc second. Distributed by NASA EOSDIS Land Processes DAAC. doi: 10.5067/MEaSUREs/SRTM/SRTMGL1.003. Accessed 2021-06-01.

23. Dmytruk, Y., Cherlinka, V. \& Demyd, I. (2019). Predicative soil cartographic materials as an element of modern large-scale surveys. Visnyk of Lviv National Agrarian University: Agronomy, 23, 202-206. doi: 10.31734/agronomy2019.01.202. [in Ukrainian]

24. latsuk, I., Dmytruk, Y., Cherlinka, V. \& Dent, D. (2021). Status and Problems of Normative Monetary Valuation of Land in Ukraine. In: Dmytruk, Y. \& Dent, D. (Eds.). Soils Under Stress: More Work for Soil Science in Ukraine. Cham: Springer International Publishing. pp. 17-26. doi: 10.1007/978-3-030-68394-8_2.

25. Hofierka, J., Parajka, J., Mitasova, H. \& Mitas, L. (2002). Multivariate interpolation of precipitation using regularized spline with tension, Transactions in GIS, 6(2), 135-150. doi: 10.1111/1467-9671.00101.

26. Cherlinka, V. R. (2019). Digital elevation models in soil science: theoretical and methodological bases and practical application: Extended Abstract of Dr. Biol. Sciences: 03.00.18. Dissertation, Chernivtsi, Yuriy Fedkovych Chernivtsi National University, 538 p. URL: https://drive.google.com/open?id=1TZubbaD3fNIk7FQUkSyZOdK_dPpozqpN [in Ukrainian]

27. Cherlinka, V. R. (2017). Influence of resolution of digital relief models on the quality of predicative simulation of soil cover. Soil Science. 18(1-2), 79-95. [in Ukrainian].

28. Cherlinka, V. R. \& Dmytruk, Y. M. (2018). Solving existing problems with soil maps in Ukraine. Biological systems, 10(1), $298-308$. URL: http://biosystemsjournal.chnu.edu.ua/index.php?journal=BioSystems\&page=article\&op=view\&path\%5B\%5D=https\%3A\%2F\%2Fdoi.org\%2F10.31861\%2F biosystems2018.01.094.

29. Cherlinka, V. (2017). Using Geostatistics, DEM and remote sensing to clarify soil cover maps of Ukraine. In: Dent, D., Dmytruk, Y. (Eds.), Soil Science Working for a Living: Applications of soil science to present-day problems. Springer-Verlag GmbH, Cham, Switzerland, Ch. 7, pp. 89-100. doi: 10.1007/978-3-319-45417-7 7.

30. Cherlinka, V. R. (2017). Variations of predictive efficiency of soil maps depending on the methods of constructing educational samples of predicative algorithms. Ecology and noospherology, 28(3-4), 55-71. URL: http://erae.dp.ua/index.php/erae/article/view/20. [in Ukrainian].

31. Shein, E. V. (2009). Granulometric composition of soils: problems of investigation methods, interpretation of results and classifications. Pochvovedenie, 3, 309-317. URL: https://elibrary.ru/item.asp?id=11722362. [in Russian].

32. Viatkin, K., Zalavskyi, Y, Bihun, O., Lebed, V., Sherstiuk, O., Plisko, I. \& Nakisko, S. (2018). Creation of the Ukrainian national soil organic carbon stocks map using digital soil mapping methods. Pochvovedenie $i$ agrohimiya, 2, 5-17. URL: https://cyberleninka.ru/article/n/sozdanie-natsionalnoy-karty-zapasov-organicheskogo-ugleroda-v-pochvah-ukrainy-s-ispolzovaniemtsifrovyh-metodov-pochvennogo. [in Russian].

33. Laktionova, T. M., Medvedev, V. V., Savchenko, K. V., Bigun, O. M., Nakisko, S. G. \& Sheiko S. M. (2012). Soil properties of Ukraine database (structure \& operating procedure). 2nd edition. Kharkiv: Apostrof. 150 p. URL: http://www.issar.com.ua/uk/vydannya/baza-dannyh-svoystva-pochv-ukrayny-struktura-y-poryadok-yspolzovanyya [in Russian].

34. Smith, J. O., Smith, P., Wattenbach, M., Gottschalk, P. I. A., Romanenkov V. A., Shevtsova, L. K., ... Lisovoi, N. V. (2007). Projected changes in the organic carbon stocks of cropland mineral soils of European Russia and the Ukraine, 1990-2070. Global Change Biology, 13(2), 342-356. doi: 10.1111/j.1365-2486.2006.01297.x.

35. FAO. (2021). The EX-Ante Carbon-balance Tool (EX-ACT). Economic and Policy Analysis of Climate Change. URL: https://www.fao.org/in-action/epic/ex-act-tool/suite-of-tools/ex-act/en.

УДК 631.4:551.4:004.942

\title{
Проблеми та можливості моделювання потенціалу секвестрації діоксиду карбону грунтами України
}

\author{
В. Р. Черлінка ${ }^{* 1,2}$, Ю. М. Дмитрук ${ }^{1}$, В. І. Собко ${ }^{3}$, М.В.Гунчак ${ }^{3}$, Т. І. Балан ${ }^{1}$, Л. В. Черлінка ${ }^{1}$
}

1 Чернівецький національний університет ім. Ю. Федьковича, Чернівці, Україна

${ }^{2}$ EOS Data Analytics inc, Menlo Park CA, USA

ЗЧернівецька фрілія ДУ «Інститут охорони ґрунтів України», Чернівці, Україна

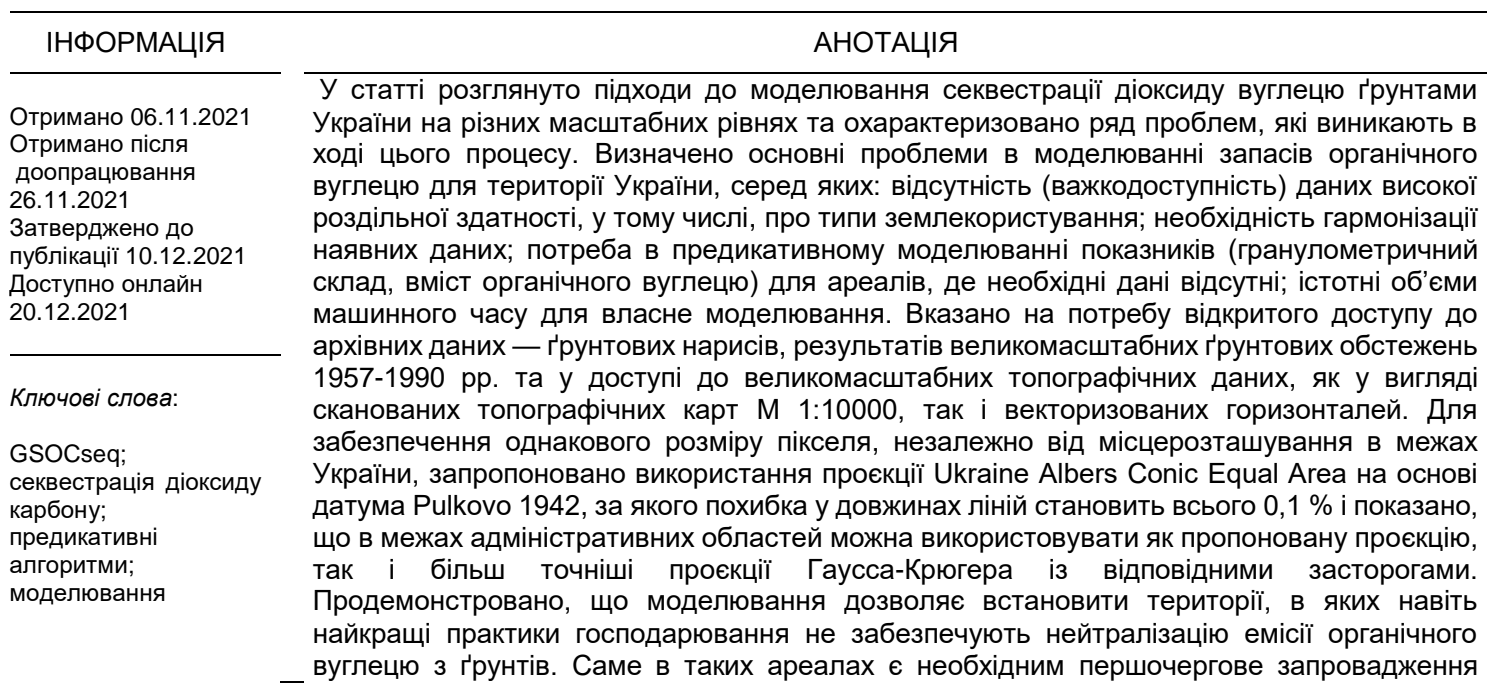



секвестрації вуглецю чи мінімізації його емісії.

E-mail: *v.cherlinka@chnu.edu.ua

Форма цитування: Cherlinka V.R., Dmytruk Y.M., Sobko V.I., Gunchak M.V., Balan T.I. \& Cherlinka L.V. (2021). Challenges and opportunities of modelling carbon dioxide sequestration potential in Ukrainian soils. AgroChemistry and Soil Science, 92, 62-70. doi:10.31073/acss92-07

УДК 631.4:551.4:004.942

\title{
Проблемы и возможности моделирования потенциала секвестрации диоксида карбона почвами Украины
}

\author{
В.Р. Черлинка ${ }^{* 1,2}$, Ю.М. Дмитрук', В.И. Собко ${ }^{3}$, М.В.Гунчак ${ }^{3}$, Т. И. Балан ${ }^{1}$, Л. В. Черлинка ${ }^{1}$
}

1 Черновицкий национальный университет им. Ю. Федьковича, Черновцы, Украина

${ }^{2}$ EOS Data Analytics Inc, Menlo Park CA, USA

${ }^{3}$ Черновицкий филиал ГУ «Институт охраны почв Украины», Черновцы, Украина

${ }^{\star} E$-mail: v.cherlinka@chnu.edu.ua

В статье рассмотрены подходы к моделированию секвестрации диоксида углерода почвами Украины на разных масштабных уровнях и охарактеризованы ряд проблем, возникающих в ходе этого процесса. Определены основные проблемы в моделировании запасов органического углерода для территории Украины, среди которых: отсутствие (труднодоступность) данных высокого разрешения, в том числе, о типах землепользования; необходимость гармонизации имеющихся данных; потребность в предикативном моделировании показателей (гранулометрический состав, содержание органического углерода) для ареалов, где необходимые данные отсутствуют; существенные объемы машинного времени для собственно моделирования. Указано на необходимость открытого доступа к архивным данным (почвенным очеркам) результатов крупномасштабных почвенных обследований 1957-1990 гг. Для обеспечения одинакового размера пикселя, независимо от местоположения в пределах Украины, предложено использование проекции Ukraine Albers Conic Equal Area на основе датума Pulkovo 1942, при котором погрешность в длинах линий составляет всего 0,1% и показано, что в пределах административных областей можно использовать как предлагаемую проекцию, так и более точные проекции Гаусса-Крюгера с соответствующими предосторожностями. Продемонстрировано, что моделирование позволяет установить территории, где даже лучшие практики хозяйствования не обеспечивают нейтрализацию эмиссии органического углерода из почв. В таких ареалах необходимо первоочередное введение адаптивного управления почвенными ресурсами агроландшафтов, которое будет способствовать секвестрации углерода или минимизации его эмиссии.

Ключевые слова: GSOCseq; секвестрация диоксида карбона; предикативные алгоритмы; моделирование.

Форма цитирования: Cherlinka V.R., Dmytruk Y.M., Sobko V.I., Gunchak M.V., Balan T.I. \& Cherlinka L.V. (2021). Challenges and opportunities of modelling carbon dioxide sequestration potential in Ukrainian soils. AgroChemistry and Soil Science, 92, 62-70. doi:10.31073/acss92-07 